\title{
Safety Concerns Regarding the use of Dental Lasers
}

\author{
Simarpreet Singh, Daljit Kapoor, Ramandeep Singh Gambhir, Amarinder Kaur, Gurminder Singh
}

\begin{abstract}
The laser technology offers a wide range of uses in dentistry with certain advantages to the general dental practitioner like bloodless surgery, minimal postoperative pain, reduction of operative time and high patient acceptance. Patient acceptance has also been demonstrated in various studies. Despite these major advantages, safety regarding the use of lasers cannot be neglected. It has become an important concern in this modern era of dentistry because of the growing application of the technology. Potential hazards can be encountered while using lasers like the ocular hazards, tissue injury, inhaling the vapors emitted by the laser procedure, fire, explosion hazards, etc. The safe use of lasers should be ensured by the individuals who might be exposed either deliberately or accidently while using lasers. Effective measures should also be undertaken by clinicians and health professionals to minimize the injuries caused due to laser accidents. This article explores the risks involved in the use of lasers in dentistry and suggests some of the laser safety protocols/measures that can be established in the dental office for prevention of laser injuries.
\end{abstract}

Keywords: Laser, Safety, Hazards, Dentistry, Measures.

How to cite this article: Singh S, Kapoor D, Gambhir RS, Kaur A, Singh G. Safety Concerns Regarding the use of Dental Lasers. Int J Laser Dent 2012;2(2):35-40.

Source of support: Nil

\section{Conflict of interest: None}

\section{INTRODUCTION}

Light has been used as a therapeutic agent for many centuries. In ancient Greece, sunlight was used in 'heliotherapy' that is the exposure of body to the sun for the restoration of health. The Chinese also used sunlight to treat conditions, such as rickets, skin cancer and even psychosis. This use of light for treatment of various pathologies is referred to as 'phototherapy'. ${ }^{1}$ Around 1400 BC, Indians used a drug called 'Psoralea' to treat vitiligo. A lotion of this drug was applied to the skin, which was then exposed to the sun. Egyptians also used Psoralea to treat leukoderma. ${ }^{2}$

The dental lasers of today have benefited from decades of laser research and have revolutionized several areas of treatment in the last three and a half decades of the 20th century. Einstein's atomic theories on controlled radiation can be credited for the foundation of laser technology. His article on stimulated emission of radiant energy is acknowledged as the conceptual basis for amplified light. ${ }^{3}$ The first laser or 'MASER' (microwave amplification by stimulated emission of radiation) as it was initially called was developed by Theodore H. After Maiman in 1960, ${ }^{4}$ there has been great interest among dental practitioners, scientists and patients to use this tool to make dental treatment more pleasant. The interaction of laser radiation on soft tissue enables dry and bloodless surgery, minimal postoperative swelling and scarring, and minimal postoperative pain. ${ }^{5}$ Lasers when used on hard tissues, encourages efficient diagnosis of caries, improves the resistance of dental enamel to caries, laser etching of enamel, cavity preparations, photopolymerization of composite resin and sterilization of the root canal system. ${ }^{6-8}$

Safe use of lasers is one of the most important concerns in the use of laser therapy. With the availability, utilization and development of different laser wavelengths and methods of pulsing, interest is developing in this growing field. ${ }^{9}$ Diodes, Nd: YAG, erbium and $\mathrm{CO}_{2}$ lasers are class 4 lasers, which are considered high-powered dental lasers. They are hazardous to eyes and skin and require special precautions. Although many regulations and standards relating to laser safety are in effect, there continues to be an average of 35 laser injuries per year in United States. ${ }^{10}$ This can be attributed to unmonitored use of lasers in many solo practices. Furthermore, the level of training and experience of dental staff is generally far less than that of the laser surgical nurse or hospital laser safety officer. ${ }^{11}$ Therefore, the focus of the present review is on the various hazards that can be encountered by the patients and the dentist while using laser and control measures that can be adopted to minimize the injuries caused by the same.

\section{LASER HAZARD CLASSIFICATION}

A hazard is something with the potential to cause injury. There are a number of hazards associated with laser use in a clinical environment, the most obvious being the laser light itself. Accidental exposure could be caused by a misaligned or misdirected laser beam, laser light escaping from the protective housing of the unit, or a broken or detached optical fiber. The Center for Devices and Radiological Health (CDRH) of Food and Drug Administration (FDA) of USA sets forth the standards governing the manufacture of lasers in the Code of Federal Regulations (CFR). ${ }^{12}$ This standard categorizes all the laser devices into one of the four classes based on their total energy output and wavelength. The lasers used in dentistry generally fall into the class IV category, which is considered to be the most hazardous group of lasers (Table 1). 
Table 1: Classification of lasers and associated hazards

Class Description

I Low powered, safe to view

Ila Low powered, visible, cannot produce any known eye or skin injury during operation based on a maximum exposure time of 1,000 seconds.

IIb Low powered, visible, incapable of emitting laser radiation at levels that are known to cause skin or eye injury within the time period of the human eye aversion response $(0.25$ $\mathrm{sec}$ ).

IIla Medium powered, visible, harmful, if viewed less than 0.25 seconds without magnifying optics.

IIIb Medium powered, hazardous, if viewed directly.

IV High powered ( $>0.5 \mathrm{~W})$, ocular, skin and fire hazards (e.g. lasers used in dentistry).

The Laser Institute of America, which serves as a secretariat for the American National Standard Institute (ANSI), has developed both general and clinical standards that currently serve as voluntary guidelines for the safe use of lasers in dentistry and medicine. ${ }^{13}$ In addition to ANSI, detailed guidelines have been published by occupational safety and health administration (OSHA). The types of hazards that may be encountered within the clinical practice of dentistry may be grouped as in Table 2 .

Table 2: Various hazards encountered in dentistry Laser hazards encountered in dentistry

1. Ocular hazards

2. Tissue damage

3. Respiratory hazards/environmental hazards

4. Combustion hazards (fire and explosion)

5. Electrical hazards (shock)

\section{Ocular Hazards}

Potential injury to the eye can occur either by direct emission from laser or from the reflection from the mirror-like surface. Dental instruments have been capable of producing reflections that may result in tissue damage to both the operator and the patient. The use of carbonized or nonreflective instruments has been recommended during laser treatment. ${ }^{13}$ Several structures of the eye may be injured as a result of laser emissions. The site of injury is directly dependent on the preferential absorption of various wavelengths by specific structures of the eye. The primary ocular injury that may result from a laser accident is a retinal or corneal burn. Retinal injury is possible with emissions in the visible and near-infrared spectral regions. Even low intensity beams can cause damage because of the focusing effect of the lens and cornea. Approximately 95\% of the incident radiation entering the eye is absorbed by pigmented epithelium of the retina and choroids layer. Irreversible retinal burns resulting in permanent blindness can occur by conversion of incident radiation to heat energy within a fraction of a second. Other potential ocular injuries that may occur from various wavelengths are, e.g. injury to the sclera, aqueous humor and cataract, etc. ${ }^{14}$

\section{Tissue Damage}

Laser-induced damage to the skin and other non-target tissue (oral tissue) can result from thermal interaction of radiant energy with tissue proteins. Temperature elevations of $21^{\circ} \mathrm{C}$ above normal body temperature $\left(37^{\circ} \mathrm{C}\right)$ can produce cell destruction by denaturation of cellular enzymes and structural proteins, which interrupts basic metabolic processes. ${ }^{14}$ Histologically, the thermal effect of absorbed radiant energy is manifested as thermal coagulation necrosis for wavelengths above $400 \mathrm{~nm}$. Other non-thermal tissue interactions are thought to induce injury through photochemical and photoacoustic mechanisms. They occur with single or repetitive pulses of very soft duration. Although there have been no reports of laser-induced carcinogenesis to date, the potential for mutagenic changes have been questioned. The potential damage caused to the deeper tissues from penetration of specific wavelengths, such as the continuous wave Nd:YAG laser is of clinical significance. ${ }^{15,16}$ With prolonged exposures of low power density from this type of laser, excess thermal necrosis can occur that may not be apparent at the tissue surface.

\section{Respiratory/Environmental Hazards}

Another class of hazards involves the potential inhalation of air borne biohazardous materials that may be released as a result of surgical application of lasers. These secondary hazards belong to a group of 'potential laser hazards' (also called as 'non-beam hazards'). They do not pertain to injuries resulting from direct exposure to laser beam. Inhaled air borne contaminants can be emitted in the form of smoke or plume generated through the thermal interaction of surgical lasers through tissue or through the accidental escape of toxic chemical and gases from the laser itself. ${ }^{17}$ Toxic gases and chemicals are a more common hazard in dental research facilities and laboratories. Some of the toxic gases and chemicals are-fluorine, hydrochloride gases, toxic dyes and solvent.

A study by the National Institute for Occupational Safety and Health (NIOSH) evaluated the air that operating room (OR) personnel were exposed to during laser procedures and found that detectable levels of ethanol, isopropanol, anthracene, formaldehyde, cyanide and airborne mutagenic particles were found. Inhalations of these toxic aerosols have been found to be potentially damaging to the respiratory system. ${ }^{18,19}$ Ablation of the infectious tissue poses an even 
greater hazard due to the possible presence within the plume of viable intact infectious agents like HIV. ${ }^{20}$

\section{Combustion Hazards}

In the presence of flammable materials, lasers may pose other significant hazards. Flammable solids, liquids and gases used within the dental surgical setting can be easily ignited, if exposed to the laser beam. Toxic fumes released as a result of combustion of flammable materials presents an additional hazard. ${ }^{21,22}$ The use of flame-resistant materials and other precaution is, therefore, recommended. Some of the common flammable materials found in the dental treatment areas are:

- Solids: Clothing, paper products, plastic, waxes and resins.

- Liquids: Ethanol, acetone, methyl methacrylate, solvents.

- Gases: Oxygen, nitrous oxide, general anesthetics, aromatic vapors.

\section{Electrical Hazards}

Most laser systems involve high potential, high current electrical supplies. The most serious accidents with lasers have been electrocutions. There are several associated hazards that may be potentially lethal. ${ }^{23}$ Electrical hazards are grouped as:

- Shock hazards

- Fire hazards or explosion hazards

Safe manufacturing practices offer adequate protection from these hazards. Insulation, shielding, grounding and housing of high-voltage electrical components provide adequate protection under most circumstances from electrical injury. Installation and servicing of laser equipment should always be performed by qualified personnel and not by the dentist. It is a good practice to have at least two persons in an area while working on high energy power systems. In laboratories, where laser power supplies are opened or serviced by laboratory personnel, dental and other auxiliary staff should be trained in cardiopulmonary resuscitation. ${ }^{24}$

\section{LASER HAZARD CONTROL MEASURES}

According to OSHA guidelines and ANSI standards, for the safe use of lasers in dentistry, control measures are required to reduce the possibility of unwanted exposure of patient and personnel to laser radiation. ${ }^{25}$ Four categories of control measures are:

1. Engineering controls

2. Personal protective equipment
3. Administrative and procedural controls

4. Environmental controls.

\section{Engineering Controls}

Engineering controls are normally designed and built into the laser equipment to provide safety. Engineering controls such as enclosures, interlocks and beam stops are very effective at eliminating hazards (if not defeated). Most engineering controls are provided in place by the laser manufacturer and hence, the user does not need to think about them. Some of the important engineering controls recommended by the ANSI are as follows. ${ }^{26}$

- Protective housing: A laser should have an enclosure around it that limits the access to the laser beam or radiation at or below the applicable maximum permissible exposure (MPE) level. A protective housing is required for all the classes of lasers except, of course, at the beam aperture.

- Master switch control: All class IV lasers (including dental lasers) and laser systems require a master switch control. The switch can be operated by a key or a computer code. When disabled (when the key or code is removed), the laser cannot be operated.

- Optical viewing system safety: Interlocks, filters or attenuators are to be incorporated in conjunction with beam shutters when optical viewing systems, such as telescopes, microscopes, viewing ports or screens are used to view the beam or beam-reflection area.

- Beam stop or attenuator: Class IV lasers require a permanently attached beam stop or attenuator which can reduce the output emission to a level at or below the appropriate maximum permissible exposure (MPE) level when the laser system is on 'standby'.

- Laser activation warning system: An audible tone or a bell and/or a visual warning (such as a flashing light) is recommended as an area control for class III b laser operation. Such a warning system is mandatory for class IV lasers.

\section{Personal Protective Equipment}

All the people within the dental treatment room must wear adequate eye protection, including the patient. When selecting the protective eyewear, several factors should be considered. They are as follows. ${ }^{25}$

- Wavelength of laser emission

- Maximum permissible exposure limits

- Degradation of absorbing media or filter

- Optical density of the eyewear

- Radiant exposure limits 
- Need for corrective lenses

- Multiple wavelength requirements

- Restriction of peripheral vision

- Comfort and fit.

Optical density is one of the most important factors to be considered while choosing the laser eye protection. The attenuation should be used to reduce the beam exposure to the eye to relatively safe levels. Laser protective eyewear is intended to provide a level of protection that may be used to stare directly into the beam. Eyewear must comply with The Personal Protective Equipment at Work Regulations SI 1992/2966 (HMSO 1992) and the British Standard BS EN 207:2009 (BSI 2010). ${ }^{27}$

\section{Administrative and Procedural Controls}

If general anesthesia is administered during any dental procedure, in place of the standard PVC intubation tube, a red rubber or silastic tube should be used. For further protection, the tube can be wrapped with $1 / 3$ to $1 / 2$ inch aluminum tape. Highly reflective instruments and those with mirrored surfaces should be avoided since they cause damage to the non-target tissues. ${ }^{14} \mathrm{~A}$ wax spatula or periosteal elevator can be inserted into the gingival sulcus to serve as an effective shield when lasing soft tissue near teeth. ${ }^{28}$

For most applications it may be advisable to use low power time settings initially before progressing to higher and faster times. When lasers are not actually been used for treatment or if long pauses occur between use, the unit should be placed in a standby mode to prevent inadvertent firing of the laser beam. Most manufacturers provide a cover or metal hood to prevent accidental activation of the laser beam. The foot switch should be cleaned and inspected prior to use to avoid getting stuck in a position while operating. Most laser accidents and injuries can be prevented, if appropriate control measures are recognized and implemented. ${ }^{13}$

\section{Environmental Controls}

Evaluation of environmental hazards involves an assessment of three primary aspects of the laser treatment area that should be considered to establish adequate control measures for the particular application, these include: ${ }^{13}$

\section{Physical Environment in which the Laser is used}

Laser use should be confined to controlled areas with restricted access. Use of protective laser curtains should be considered to prevent accidental exposures to passers-by. Fail-safe mechanisms that prohibit the laser from firing when doors are opened are also useful to prevent accidental exposure to persons entering the operating room during laser procedures. ${ }^{29,30}$ All entrances to the operating theater should be clearly marked with a removable warning signs that contains the words 'Danger' and 'Laser Radiation'. Highly reflective instruments and surfaces should be avoided to prevent reflection of the laser beam onto the non-target tissues.

\section{Potential for Injury Attributed to Direct Exposure from the Laser Beam Output and Delivery Mechanism}

To avoid an electrical hazard during the operation of the laser unit, the floor of the operating room must be kept dry. Because laser energy generates heat, care must be taken to avoid the use of flammable and explosive liquid or gases in the operating room. Flammable materials, such as surgical drapes and gauze sponges may be soaked in sterile saline to reduce the potential of burning by accidental exposure to the laser beam. ${ }^{29}$

\section{Level of Training and Knowledge of Laser Safety of the Persons}

All staff members should receive objective and recognized training in the safety aspects of laser use within dentistry, as with other specialties. ${ }^{30}$ The ANSI Z136.1 standard states that the management (employer) has the fundamental responsibility for the assurance of the safe use of lasers owned and/or operated by the employer. ${ }^{26}$ There are three main types of training programs:

- Laser safety awareness

- Laser safety refresher

- Laser safety update.

\section{Role of Laser Safety Officer}

The laser safety officer (LSO) is an individual designated to be responsible for a laser or system of lasers and for the preparation and enforcement of a safety plan, including standard operating procedures for the safe operation of lasers. The LSO has the authority and responsibility to monitor and enforce the control of laser hazards and to effect the knowledgeable evaluation and control of laser hazards. ${ }^{12}$ Dental practices offering class IIIb and IV laser treatment, must appoint a laser protection advisor (LPA) and a LSO. The LPA is usually a medical physicist who will advise on the protective devices required, MPE and nominal ocular hazard distance (NOHL) for any given wavelength being used. The LSO is appointed to ensure that all safety aspects of laser use are identified and enforced. Ideally, this could be a suitably trained and qualified dental surgery assistant. ${ }^{11,31}$ 
According to ANSI, the LSO and other employees routinely working with or around lasers are strongly recommended to participate in laser safety training programs.

\section{CONCLUSION AND RECOMMENDATIONS}

Dentistry has entered the 21st century, an era of high technology. The dental laser has opened new vistas for the dentist into the high-technology dentistry. At present, only very few applications of laser treatment have become well accepted methods. Safety regarding the use of lasers is not optional. It should be the highest priority of all the staff members involved in the surgical procedure. There should be a concerted effort to better educate physicians, provide more educational support for perioperative staff members, and conduct additional research to improve equipment and ancillary supplies. Prevention of accidents requires thorough knowledge of their causes and the application of measures to avoid them. Based on the hazards encountered due to lasers and various control measures that can be undertaken to prevent injuries, the following recommendations are put forth: ${ }^{32,33}$

1. All class IV lasers (most hazardous lasers) should be considered potentially hazardous to anyone present during the time their operation.

2. It is strongly advised that any safety precautions delineated by the manufacturer in the operator's manual and general safety precautions listed within the ANSI and OSHA standards should be strictly followed.

3. Prior to any clinical or research application of a laser, the intended operator of the laser system must have a thorough knowledge of the operation and safety requirements of the specific laser system and should have received hands-on instructions related to its practical application.

4. All the staff or other personnel present within the laser operating field should be well versed in the general safety practices applicable to the laser operation.

\section{REFERENCES}

1. Daniell MD, Hills JS. A history of photodynamic therapy. Aust NZ J Surg 1991;61:340-48.

2. Mukerji B. Psoralea and other indigenous drugs used in leucoderma. J Sci Ind Res 1956;15A:1-12.

3. Bailoor DN, Nagesh KS. Fundamentals of Oral Medicine and Radiology (1st ed), New Delhi: Jaypee Brothers Medical Publishers, 2005.

4. Miserendino LJ, Pick RM. Lasers in dentistry (1st ed). Quintessence Publishing Co 1995,17-25.

5. Lomke MA. Clinical applications of dental lasers. Gen Dent 2009;57(1):49-59.
6. Slavoljub Z, Larisa B, Mila K. Lasers in Dentistry. Serbian Dental J 2004;51:146-52.

7. Clarkson DM. Lasers in dentistry. Dent Update 1992;19(3): 115-16.

8. Walsh LJ. The current status of laser applications in dentistry. Aust Dent J 2003;48(3):146-55.

9. Wigdor HA, Walsh JT, Featherstone JDB, Visuri SR, Fried D, Waldvogel JL. Lasers in dentistry. Lasers Surg Med 1995;16: 103-33.

10. Sweeney C. Laser Safety in dentistry. Gen Dent 2008;56(7): 660-1, 767.

11. Parker S. Laser regulation and safety in general dental practice. Br Dent J 2007;202(9):523-32.

12. Mastis R. A Primer for the Laser Safety Officer. J Laser Dent 2011;19(1):168-71.

13. Laser safety manual [homepage on the internet]. Laser Institute of America; 2005 [cited 2012 April 26]. Available from: www.laserinstitute.org

14. Neiburger EJ, Miserendino L. Laser reflectance: Hazard in the dental operatory. Oral Surg Oral Med Oral Pathol 1988;66: 659-61.

15. Berk G, Atici K, Berk N. Treatment of gingival pigmentation with Er, Cr:YSGG Laser. J Oral Laser Applications 2005;5: 249-53.

16. Pick RM, Coluard MD. Current status of laser in soft tissue dental surgery. J Periodontol 1993;64:589-602.

17. Koksa JM, Eugene J. Chemical composition of laser-tissue interaction smoke plume. J Laser Appl 1989;3:59-63.

18. Barrett WL, Garber SM. Surgical smoke: A review of the Literature. Business Briefing: Global Surgery 2004:1-7.

19. Freitag L, Chapman GA, Sielczak M, Ahmed A, Russin D. Laser smoke effect on the bronchial system. Lasers Surg Med 1987; 7:283-88.

20. Baggish MS, Poiesz BJ, Joret D, Williamson P, Refai A. Presence of human immunodeficiency virus DNA in laser smoke. Lasers Surg Med 1991;11(3):197-203.

21. Davis RK, Simpson GT. Safety with the carbon dioxide laser. Otolaryngol Clin North Am 1983;16:801-14.

22. Lim RY, Kenney CL. Precaution and safety in carbon dioxide laser surgery. Otolaryngol Head Neck Surg 1986;95(2):239-41.

23. Dudelzak J, Goldberg DJ. Laser safety. Curr Probl Dermatol 2011;42:35-39.

24. Marshall WJ. Update of national standards for the safe use of lasers outdoors. J Laser Appl 1999;11:234-36.

25. Miserendino LJ, Abt E, Harris D, Wigdor H. Recommendations for safe and appropriate use of lasers in dentistry in face of rising concerns. J Laser Appl 1992;4(3):16-17.

26. American National Standards Institute, American National Standard for the Safe Use of Lasers, Laser Institute of America: New York; ANSI Z; 1993. ANSI Z Publication 136.1.

27. Mary S. Laser safety: Practical measures and latest legislative requirements. J Perioper Pract 2011;21(9):299-303.

28. Schuller DE. Use of the laser in the oral cavity. Otolaryngol Clin North Am 1990;23(1):31-42.

29. Andersen K. Safe use of lasers in the operating room-what perioperative nurses should know. AORN J 2004;79:171-88.

30. Szymanska J. Work-related vision hazards in the dental office. Ann Agric Environ Med 2000;7:1-4.

31. Rice JH. Laser safety officer: A vital role for dental assistants. Dent Assist 2005;74(1):26-27. 
32. Piccione PJ. Dental laser safety. Dent Clin North Am 2004;48(4):795-807.

33. Bargman H. Laser safety guidelines. J Clin Aesthet Dermatol 2010;3(5):18-19.

\section{ABOUT THE AUTHORS}

\section{Simarpreet Singh}

Associate Professor and Head, Department of Public Health Dentistry Gian Sagar Dental College and Hospital, Rajpura, Punjab, India

\section{Daljit Kapoor}

Professor and Head, Department of Periodontics, Gian Sagar Dental College and Hospital, Rajpura, Punjab, India

\section{Ramandeep Singh Gambhir (Corresponding Author)}

Assistant Professor, Department of Public Health Dentistry, Gian Sagar Dental College and Hospital, Rajpura-140601, Punjab, India Phone: +91-99156-46007, Fax: +91-1762 520011, e-mail: raman1g@yahoo.co.in

\section{Amarinder Kaur}

Assistant Professor, Department of Oral Medicine and Radiology Gian Sagar Dental College and Hospital, Rajpura, Punjab, India

\section{Gurminder Singh}

Professor, Department of Prosthodontics, Gian Sagar Dental College and Hospital, Rajpura, Punjab, India

\section{Corrigendum}

The article—-Root Coverage with a Free Gingival Autograft using an Er,Cr:YSGG Laser: A Case Report by Dr. Deepalakshmi Damodaran and Mrs. Pratebha Balu was mistakenly printed without pictures in the International Journal of Laser Dentistry, January-April 2012, Volume 2, Number 1. We sincerely apologize for the inconvenience caused. The soft copy of the article is now available with the photos on the following link: http://www.jaypeejournals.com/eJournals/ShowText.aspx?ID=2736\&Type=FREE\&TYP=TOP\&IN= / eJournals/images/JPLOGO.gif\&IID=215\&isPDF=YES 